\title{
AUTOMATED FACE DETECTION AND TRACKING FACIAL FEATURES IN VIDEOS
}

\author{
Renuka Devi M.N \\ Department of CSE \\ Dayananda Sagar University, Bengaluru, Karnataka, India
}

\begin{abstract}
In our paper we propose efficient face detection and tracking system that utilizes facial landmark features as key features of the face implemented in KLT algorithm. Sequences Video Frames gives us more information than a single image. Automated detection and tracking of a face using key feature extractors (points) play a significant role in vast variety of applications including Human action recognition, Automated Surveillance system, and human computer interaction. In our methodology, we have developed simple face detection and tracking system by splitting the tracking method in to three parts. 1) Detecting a face, 2) identify facial features for tracking, 3) Track the face. Detecting the face is implemented using Viola Jones object detector, and tracking is done by KLT algorithm. Investigated results shows that our system is more robust and efficient in processing the video frames to various kinds of face rotations and occlusions.
\end{abstract}

Keywords - Face detection, Facial features, KLT algorithm, Face tracking, Viola Jones method.

\section{INTRODUCTION}

Face detection and recognition or any object detection and tracking have been a great challenge for researchers from few decades. It is an important research topic and demanding task in computer vision applications. Face detection plays a significant role in applications like biometric identifications, intelligent human computer interface, surveillance, humanrobot interaction.[1] Etc. Facial attributes detection and tracking is getting more intensified and its ease of use in security and safety related applications to categorize normal and abnormal situations across real time. These type of tracking systems are effectively used to direct and indicate the signals in machines or robots. Face Detection and tracking faces in a video is a immense research problem because of many factors and changing configurations such as angle of view, pose variations, occlusions, face orientations, background intensity and illumination factors.

Many researchers have proposed different approaches to address the problem of face detection and tracking in videos which includes 1) Knowledge based method, 2) Feature invariant approaches, 3) Template matching methods, 4) Appearance based methods. Each method possesses its advantages and drawbacks. [2, 3]. Any face detecting and tracking method will have some abnormalities which will deviate from the required object of interest. Any tracking method will be efficient and robust only if it is able to minimize the deviation from the required result and should be able to handle the challenges in processing the videos. In our paper we are using Viola-Jones method and a trained object model for detection. In order to track the face from frame to frame in video we are using Kanade-Lucas-Tomasi(KLT)[4] algorithm, since this method is more effective and accurate compared to many existing approaches.

\section{RELATED WORK}

There exists many reported research work related to automated face detection and tracing. Many methods have been Gupta and Tiwari implemented Viola Jones algorithm for face detection in images. [5]. Najdawai et al. [4] used Kalman technique to locate optimal features and detect shadow for human face. Panchal et al [5] implemented face detection algorithm using Lucas Kanade Tracker algorithm. Agarwal and Khatri [6] implemented Viola Jones algorithm to detect the face in the video frames. Success has been achieved with each method to varying degrees and complexities. In our paper, proposed methods detect the face in every single frame in a video, and then the KLT algorithm is used for tracking the facial features across the video frames. $[7,8]$ 


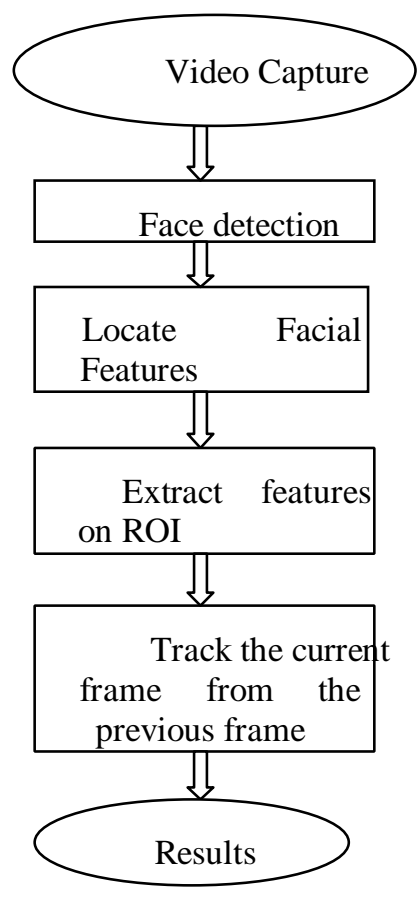

Figure1: Proposed methodology

\section{PROPOSED METHODOLOGY}

Automated detecting and tracking a face using feature points are significant in various Computer vision applications like human activity detection, human computer interaction, and automated safety surveillance system. In our paper, we propose a simple face detection and tracking method by categorizing the problem in to three parts as explained in Figure 1.

- Face detection

- Trace/extract facial features for tracking

- Track the current frame from the previous frames

\section{Face detection}

In this step, we detect the face using Cascade Object detector to identify the object of interest and locate the face in a video frame. Object detector uses Viola- Jones detection method and a trained classification model for detection. ViolaJones method institute out the positions of the human faces in video frames. The detector is designed to detect any face or object of interest in sequences of video frames.

Viola-Jones Boosting Algorithm. [9]

- Simple weak classifier is Single feature perceptron

- With K features (example: $\mathrm{K}=150,000$ ), we get 150,000 various single feature perceptron

- At every step of boosting by Viola Jones we have
- Given reweighted feature points from previous frames - Train all K features $(150,000)$ single feature perceptrons.

- Choose the best classifier at this level

- Merge with the further formerly selected classifiers.

- Reweight the data points(features)

- study all $\mathrm{K}$ classifiers again, select the excellent one, combine them and reweight it again

- Recur until we get the F classifiers preferred.

\section{Locate Facial Features to Track}

To track the face feature frame by frame, we are using Kanade-Lucas-Tomasi(KLT) algorithm. Object detectors are computationally expensive and since it cannot handle the challenges like object( face) rotation and tilt head by an angle. Hence it is used for detecting the face only, and we are using KLT algorithm for tracking set of interested feature points across the video frames.

Once the face is located, the algorithm should automatically identify the feature points having sufficient texture which can be consistently tracked. In our paper, the method used detects the feature points which are tracked that minimizes the SSD dissimilarity between windows centered at current feature point position. Which has sufficient texture for automatic tracking. The key features are extracted for detection of the face. Next, the first frame is traced from previous one using Vision.PointTracker() to identify the features of the face. Then, Then estimate Geomertic Transform () function implemented to approximate the revolution, transformation and range between current feature points and previous feature points. It is also applied to the bounding box around the face in video frames.

In our subsequent step we are developing a point tracker and enable the bidirectional error constraint to make it more robust in the presence of noise and clutter. Later we are also creating a video player object for displaying video frames. The KLT algorithm is still extensively used compared to other methods since it fully automated method and its performance is competitive in terms of feature point quality.

\section{Track the face}

\section{KLT algorithm for tracking [ 10]}

Functions: are used to select and track good features from frame to frame(KLT-identify-Good-Features, KLT-TrackFeatures) to estimate the movement of face. Modify the tracker and use geometric transform to detect the key points.

Structures: KLT_Tracking-background, KLT_Features, KLT_FeatureList. $\quad$ Gradients are computed in $\mathrm{x}$ and $\mathrm{y}$ way 


\section{International Journal of Engineering Applied Sciences and Technology, 2020 \\ Vol. 5, Issue 7, ISSN No. 2455-2143, Pages 148-151 \\ Published Online November 2020 in IJEAST (http://www.ijeast.com)}

and are used to choose the features $\square$ pc-missedPixels can be used to fasten the steps.

Integrity of pixel deliberated as minimum Eigen value of gradient matrix

- Pixels are sorted in ascending order of integrity

- mFeatures are selected

- Assemble mindistance and minimum_eigen value.

\section{EXPERIMENTAL RESULTS}

We have considered videos from ted talks and also videos from VIT data base for multiple face detection and tracking in videos. Figure 2,3 and 4 from ted talks, where our algorithm is able to detect and track the face when tilted and rotate by an angle. In figure 3 , we have multiple (four) faces detected and tracked.
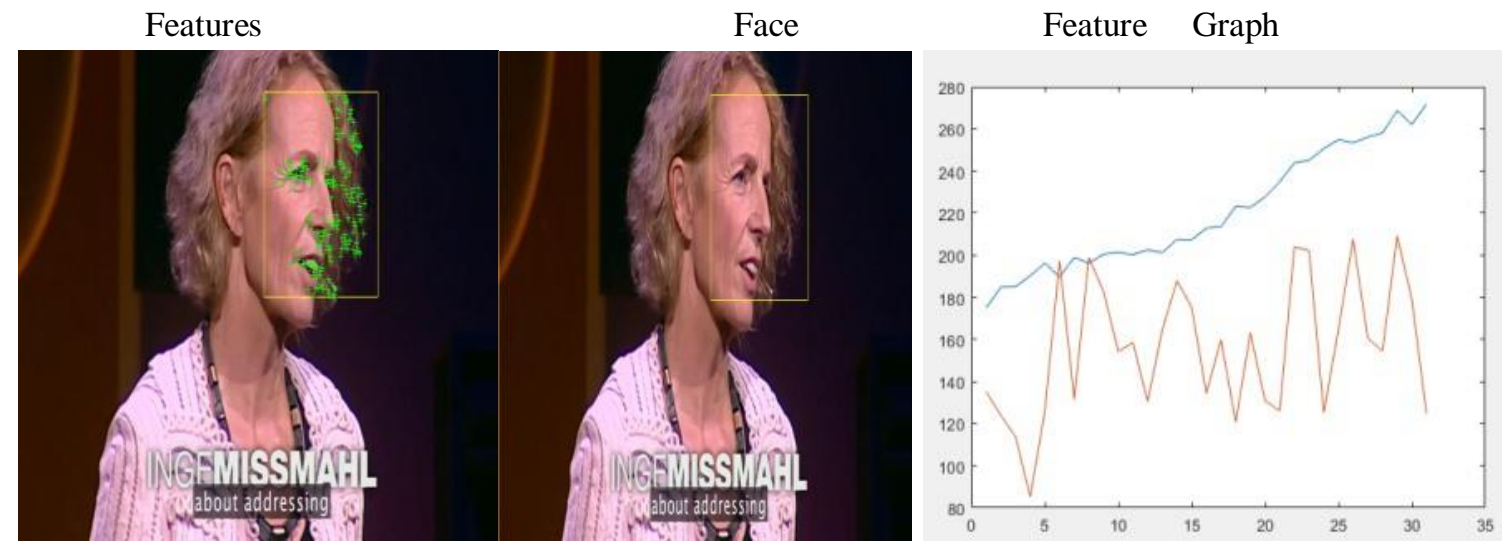

Figure 2: Face detection and tracking with head tilt pose with feature graph

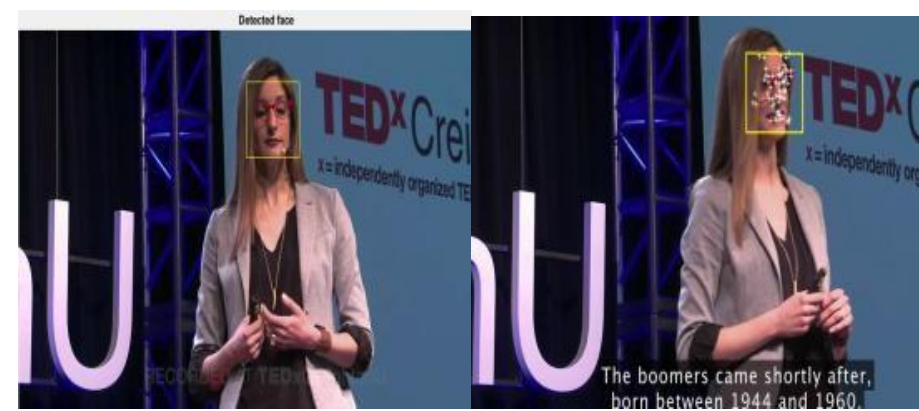

Figure3: Face detection and tracking (direct face pose)

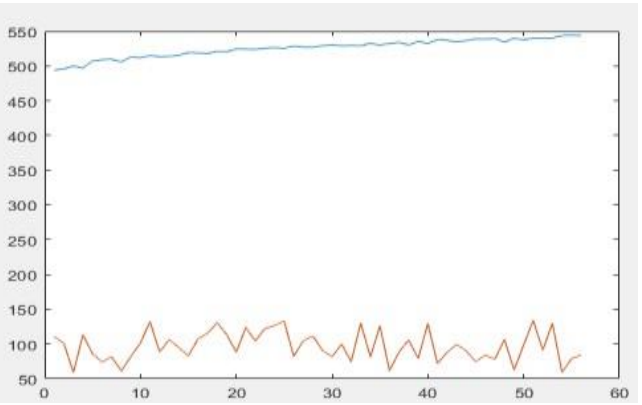

(Direct face pose)
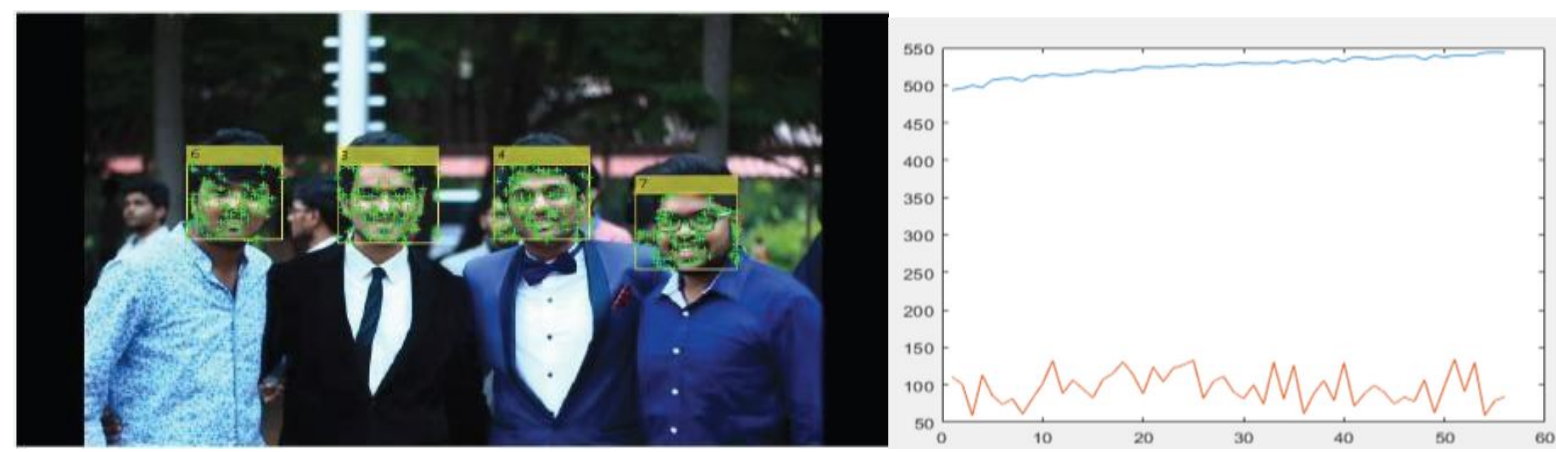

Figure 4: Multiple face detection from VIT database video 
Table1: Accuracy for face detection

\begin{tabular}{|c|c|c|c|}
\hline Videos & $\begin{array}{c}\text { Total no of } \\
\text { features } \\
\text { identified }\end{array}$ & $\begin{array}{c}\text { Total number } \\
\text { of non } \\
\text { facial } \\
\text { features }\end{array}$ & Accuracy(\%) \\
\hline 1 & 40 & 5 & 89 \\
\hline 2 & 35 & 9 & 82 \\
\hline 3 & 38 & 2 & 92 \\
\hline 4 & 55 & 3 & 94 \\
\hline
\end{tabular}

\section{CONCLUSIONS}

The proposed method presented in our paper for automated face detection and tracking reduces estimated computation time resulting with high accuracy compared to other methods. Viola -Jones cascaded object detector used for face detection and KLT algorithm used for tracking of faces in sequence of video frames.

Most of the security and surveillance systems, video structuring and indexing requires can be developed in real time and required object can be tracked robustly. Our method in the paper can handle multiple faces in a single frame, as well as changing scale and pose. Experiments carried out on a large number of data sets taken from different ted talk videos and other various datasets; show a clear improvement over the results of feature-based detection.

\section{REFERENCES}

[1] Danelakis, Antonios, Theoharis Theoharis, and Ioannis Pratikakis. (2018) "Action unit detection in 3D facial videos with application in facial expression retrieval and recognition." Multimedia Tools and Applications: 1-29.

[2] Mahmood, Zahid, et al. (2017): "A review on stateof-the-art face recognition approaches." Fractals 25.021750025 .

[3] Giannakakis, G., et al. (2017): "Stress and anxiety detection using facial cues from videos." Biomedical Signal Processing and Control 31 89-101.

[4] Ranganatha, S., et al. (2018). "Selected Single Face Tracking In Technically Challenging Different Background Video Sequences Using Combined
Features." ICTACT Journal on Image \& Video Processing9.2

[5] Sharma, J. Jayapradha Soumya, and Yash Dugar. (2018): "Detection and Recognition of Human Emotion using Neural Network." International Journal of Applied Engineering Research 13.8 64726477.

[6] Lacarrubba Flores, Maria Dora Jazmin, et al. (2018): "Femoralfacial syndrome: A review of the literature and 14 additional patients including a monozygotic discordant twin pair." American Journal of Medical Genetics Part A 176.9 1917-1928.

[7] Ranganatha, S., and Y. P. Gowramma. (2018): "Image Training, Corner and FAST Features based Algorithm for Face Tracking in Low Resolution Different Background Challenging Video Sequences." International Journal of Image, Graphics and Signal Processing 10.839.

[8] Sharma, Prateek, Pranjali M. Kokare, and Maheshkumar H. Kolekar. 2019"Performance Comparison of KLT and CAMSHIFT Algorithms for Video Object Tracking." Recent Trends in Communication, Computing, and Electronics. Springer, Singapore,. 323-331.

[9] Sun, Xudong, Pengcheng $\mathrm{Wu}$, and Steven $\mathrm{CH}$ Hoi. (2018): "Face detection using deep learning: An improved faster RCNN approach." Neurocomputing 2994250.

[10] AlQahtani, Faleh, et al. (2019): "Detection and tracking of faces in 3D using a stereo camera arrangements." International Journal of Machine Learning and Computing 9.1 35-43. 